TRANSACTIONS OF THE

AMERICAN MATHEMATICAL SOCIETY

Volume 181, July 1973

\title{
TWO-NORM SPACES AND DECOMPOSITIONS OF BANACH SPACES. II
}

BY

\author{
P. K. SUBRAMANIAN AND S. ROTHMAN \\ Dedicated to Professor Hari Shankar, Delhi University, India
}

\begin{abstract}
Let $X$ be a Banach space, $Y$ a closed subspace of $X^{*}$. One says $X$ is $Y$-reflexive if the canonical imbedding of $X$ onto $Y^{*}$ is an isometry and $Y$ pseudo reflexive if it is a linear isomorphism onto. If $X$ has a basis and $Y$ is the closed linear span of the corresponding biorthogonal functionals, necessary and sufficient conditions for $X$ to be $Y$-pseudo reflexive are due to I. Singer. To every $B$-space $X$ with a decomposition we associate a canonical two-norm space $X_{s}$ and show that the properties of $X_{s}$, in particular its $\gamma$-completion, may be exploited to give different proofs of Singer's results and, in particular, to extend them to $B$-spaces with decompositions. This technique is then applied to a study of direct sum of $B$-spaces with respect to a $B K$ space. Necessary and sufficient conditions for such a space to be reflexive are obtained.
\end{abstract}

1.0 Introduction. Given a Banach space ( $B$-space for short) $\left(X, \|_{1}\right)$ with a Schauder decomposition $\left\{X_{j}\right\}$, a two-norm space $X_{s}$, called the canonical turo-norm space of $X$, was introduced in [10] and the properties of $X$ were then studied by investigating the properties of $X_{s}$. The aim of this paper is to continue these investigations and, in particular, study the so-called pseudo reflexivity of $X$ with respect to $A\left(X_{s}\right)$, the $\gamma$-linear functionals on $X_{s}$. Some of our results generalize those of Singer [9] to the setting of Schauder decompositions providing, at the same time, a slightly different method of proof of these results.

The basic topic of our discussion is the notion of $\gamma$-completion of the canonical two-norm space. A canonical two-norm space may be defined whenever $X$ is the direct sum of subspaces for a metric topology, not necessarily the norm topology. In $\$ \$ 5-7$, we study spaces $X=B-\Sigma_{n} X_{n}$ of sequences $\left\{x_{n}\right\}$ where $x_{n}$ belongs to a $B$-space $X_{n}$ such that $\left\{\left\|x_{n}\right\|\right\}$ is an element of a given $B K$ space $B$ of real sequences. We extend the notions of boundedly complete and shrinking decompositions to this setting and obtain analogues of some of the results in Schauder decomposition theory (cf. [10]).

Received by the editors December 16, 1970 and, in revised form, August 5, 1972.

AMS (MOS) subject classifications (1970). Primary 46B15, 46B05, 46B10; Secondary ‘6A35, 46A45.

Key words and phrases. Schauder basis, Schauder decomposition, reflexive, pseudo reflexive, two-norm space, spaces with mixed topology. 
2.0 Notations and preliminaries. A (weak) decomposition $\left\{X_{j}\right\}$ for a $B$-space $(X,||)$ is a collection $\left\{X_{j}\right\}$ of nontrivial subspaces of $X$ such that to each $x$ in $X$, there corresponds a unique sequence $\left\{x_{j}\right\}, x_{j}$ in $X_{j}$, and $x=\Sigma_{j} x_{j}$ in the norm (respectively weak) topology of $X$. It is called a Schauder decomposition of $X$, if for each $j$ the projection map $P_{j}: X \rightarrow X_{j}$, defined by $P_{j}(x)=x_{j}$, is continuous. The decomposition $\left\{X_{j}\right\}$ is called boundedly complete if every sequence $\left\{x_{j}\right\}, x_{j}$ in $X_{j}$, with the property

$$
\sup _{n}\left\{\left|\sum_{k=1}^{n} x_{k}\right|\right\}<\infty
$$

defines an element of $X$, that is, $\Sigma_{j} x_{j}$ converges. The decomposition $\left\{X_{j}\right\}$ is called sbrinking for $f$ in $X^{*}$ if $|f|_{n}^{*} \rightarrow 0$ as $n \rightarrow \infty$, where

$$
|f|_{n}^{*}=\sup _{n}\left\{|f(x)|:|x| \leq 1, x \in \sum_{j>n} x_{j}\right\} \text {. }
$$

In this paper all decompositions are assumed to be monotone, that is, $\left|\Sigma_{k \leq n} x_{k}\right| \leq$ $\left|\Sigma_{k} x_{k}\right|$ for all $n$. The reader will later observe that the proofs of analogues of our theorems to the nonmonotone case present no difficulties.

A two-norm space $X_{s}=\left(X,\left\|_{1},\right\|_{2}\right)$ is a linear set $X$ with two norms \|\|$_{1}$ and $\|_{2}$. In general, the second norm $\|_{2}$ is an $F$-norm although, in most cases of interest, it is the usual (homogeneous) norm. In this paper, all two-norm spaces considered are such that $\left(X, \|_{1}\right)$ is a Banach space and $\left(X, \|_{2}\right)$ is a normed linear space. An exception occurs in $\$ 4$ where the second norm is a $B_{0}^{*}$-norm. We recall that a $B_{0}^{*}$-norm $\left.\right|_{2}$ on $X$ is such that

$$
|x|_{2}=\sum_{k} 2^{-k}\left\{[x]_{k} /\left(1+[x]_{k}\right)\right\},
$$

[]$_{k}$ being homogeneous pseudo norms such that $[x]_{k}=0$ for all $k$ implies $x=0$. A sequence $\left\{x_{n}\right\} \subset X_{s}$ is said to be $\gamma$-convergent to $x$ in $X$, written $x_{n} \stackrel{\gamma}{\rightarrow} x$, if $\left\{\left|x_{n}\right|_{1}\right\}$ is bounded and $x_{n} \rightarrow x$ in $\left.\right|_{2}$. A $\gamma$-Caucby sequence is defined analogously and $X_{s}$ is called $\gamma$-complete if it is sequentially complete for the convergence $(\gamma)$. The space $X_{s}$ is called normal if

$$
x_{n} \stackrel{y}{\longrightarrow} x \Rightarrow|x|_{1} \leq \lim _{n} \inf \left|x_{n}\right|_{1} .
$$

A $y$-linear functional on $X_{s}$ is a linear functional which is continuous for the convergence $(\gamma)$. The set of all $\gamma$-linear functionals on $X_{s}$ will be denoted by $A\left(X_{s}\right)$. We shall say that the space $X_{s}$ satisfies property $\left(n_{0}\right)$ if $\|_{2}$ is coarser than $\|_{1}$, that is, every sequence that converges to 0 in $\|_{1}$ does so in $\|_{2}$ as well.

Let $\left(X_{j}^{*},||_{j}^{*}\right)=\left(X,||_{j}\right)^{*}, j=1,2$. For a normal two-norm space satisfying $\left(n_{0}\right)$, it is known that 


$$
X_{2}^{*} \subseteq A\left(X_{s}\right) \subseteq X_{1}^{*}
$$

$A\left(X_{s}\right)$ is a closed subspace of $X_{1}^{*}$ and that $A\left(X_{s}\right)$ is the closure of $X_{2}^{*}$ in $\left(X_{1}^{*}\right.$, ||$\left._{1}^{*}\right)$. Further, $A\left(X_{s}\right)$ is norm determining, that is,

$$
\left\{x_{1}=\sup \left\{|f(x)|: f \in A\left(y_{s}\right)\right\}\right. \text {. }
$$

The space $X_{s}$ is called $\gamma$-reflexive if $\left(A\left(X_{s}\right), \|\left.\right|_{1} ^{*}\right) *=\left(A\left(X_{s}\right)^{*}, \|_{1}^{*}\right)$ is isometrically isomorphic to $\left(X,||_{1}\right)$ under the canonical map $J$, where for $x$ in $X, J(x) f=$ $f(x)$ for all $f$ in $A\left(X_{s}\right)$. Whenever $A\left(X_{s}\right)=X_{1}^{*}, X_{s}$ is called saturated.

Let $\left\{X_{j}\right\}$ be a decomposition for the $B$-space $\left(X,\left.\right|_{1}\right)$. The canonical twonorm space $X_{s}$ of $X$ is the two-norm space $\left(X,||_{1},||_{2}\right)$, where for $x=\Sigma_{j} x_{j}$ in $X$,

$$
|x|_{2}=\sum_{j}\left|x_{j}\right|_{1} / 2^{j}
$$

The norm $\|_{2}$ is called the canonical second norm. We shall continue to use the symbol $X_{1}^{*}$ for $\left(X, \|_{1}\right) *$ and $X_{2}^{*}$ for $\left(X,||_{2}\right) *$. The reader should not confuse these symbols for the duals of the subspaces $X_{1}$ and $X_{2}$ respectively. This confusion can be easily avoided if he remembers that the duals of the subspaces themselves are nowhere considered in this paper.

The basic properties of the canonical two-norm space may be found in [10]. However, for the convenience of the reader, we recall the following notions and notations that are necessary for an understanding of the present paper.

Let $\left(X,||_{1}\right)$ be a $B$-space with Schauder decomposition $\left\{X{ }_{j}\right\}, P_{j}$ the continuous projection of $X$ onto $X_{j}$ and let $X_{s}$ be the canonical two-norm space. Then $A\left(X_{s}\right)$ has the Schauder decomposition $\left\{P_{j}^{*}\left(X_{1}^{*}\right)\right\}$. To simplify notation, we shall of ten write $A\left(X_{s}\right)_{j}$ for $P_{j}^{*}\left(X_{1}^{*}\right)$. The canonical two-norm space of $A\left(X_{s}\right)$ will be referred to as the $k$-dual of $X_{s}$ and will be denoted by $k-X_{s}$. The Schauder decomposition of $A\left(k-X_{s}\right)$ will be denoted by $\left\{A\left(k-X_{s}\right)_{j}\right\}$ and the canonical two-norm space $A\left(k-X_{s}\right)_{s}$ of $A\left(k-X_{s}\right)$ by $k^{2}-X_{s}$. When $\left\{X_{j}\right\}$ is monotone, the canonical map $J$ is an isometry (with respect to both norms) of $X_{s}$ into $k^{2}-X_{s}$. When this map is also onto, one says $X_{s}$ is $k$-reflexive. Finally, we use the symbol $\Sigma^{*}$ to indicate convergence in the weak-* topology of a given dual space.

3.0 The $\gamma$-completion of $X_{s}$. Let $\left(X, \|_{1}\right)$ be a $B$-space with Schauder decomposition $\left\{X_{j}\right\}$ and canonical two-norm space $X_{s}$. As a consequence of $[10$, Theorem 3.6], it follows that $X_{s}$ may not, in general, be $\gamma$-complete. We shall now show that $X_{s}$ may be imbedded isometrically isomorphically into a $\gamma$-complete, normal two-norm space $C(X)_{s}$ such that $X_{s}$ is $\gamma$-dense in $C(X)_{s}$.

Theorem 3.1. The sets

$$
\left\{\left\{J\left(x_{j}\right)\right\}: x_{j} \in X_{j}, \sup _{n}\left[\left|\sum_{j \leq n} J\left(x_{j}\right)\right|_{1}^{* *}\right]<\infty\right\}
$$


and

are identical.

$$
\left\{\left\{J\left(x_{j}\right)\right\}: \sum_{j}^{*} J\left(x_{j}\right) \text { converges in } \sigma\left(A\left(X_{s}\right)^{*}, A\left(X_{s}\right)\right)\right\}
$$

Proof. Consider $\left\{J\left(x_{j}\right)\right\} \subset A\left(X_{s}\right) *$ such that $\sup _{n}\left|\Sigma_{j \leq n} J\left(x_{j}\right)\right|_{1}^{* *}$ is finite. Since $A\left(X_{s}\right)$ is the norm-closure of the set $\bigcup_{k} P_{k}^{*}\left(X_{1}^{*}\right)$ in $X_{1}^{*}[2$, p. 277], and $\left\{\Sigma_{k \leq n} J\left(x_{k}\right)\right\}$ converges pointwise on $\bigcup_{k} P_{k}^{*}\left(X_{1}^{*}\right)$, it follows by the Banach-Steinhaus theorem that $\Sigma_{k}^{*} J\left(x_{k}\right)$ converges as desired.

Conversely the convergence of $\Sigma_{k}^{*} J\left(x_{k}\right)$ implies, by the Uniform Boundedness Principle, that $\sup _{n}\left|\Sigma_{j \leq n} J\left(x_{j}\right)\right|_{1}^{* *}$ is finite and the proof is complete.

Let $C(X)=\left\{\left\{J\left(x_{j}\right)\right\}: \Sigma_{j}^{*} J\left(x_{j}\right)\right.$ converges in $\left.\sigma\left(A\left(X_{s}\right) *, A\left(X_{s}\right)\right)\right\}$. As a consequence of Theorem 3.1, we may equip $C(X)$ with the norm \|\|$_{1}^{* *}$ defined by

$$
\left|\left\{J\left(x_{j}\right)\right\}\right|_{1}^{* *}=\sup _{n}\left|\sum_{k \leq n} J\left(x_{k}\right)\right|_{1}^{* *}
$$

and a canonical second norm $\|_{2}^{* *}$ defined by

$$
\left|\left\{J\left(x_{j}\right)\right\}\right|_{2}^{* *}=\sum_{j} \frac{\left|J\left(x_{j}\right)\right|_{1}^{* *}}{2^{j}}=\sum_{j} \frac{\left|x_{j}\right|_{1}}{2^{j}}
$$

Theorem 3.2. Let $C(X)_{s}=\left(C(X),\left\|_{1}^{* *},\right\|_{2}^{* *}\right)$. Then $C(X)_{s}$ is $\gamma$-complete, normal and the map $i$ defined for $x=\Sigma_{j} x_{j}$ in $X$ by $i(x)=\left\{J\left(x_{j}\right)\right\}$ is a linear isomorphism of $X$ into $C(X)$. Further, $i$ is an isometry with respect to both norms and $i\left(X_{s}\right)$ is $\gamma$-dense in $C(X)_{s}$.

Proof. Let $\left\{p_{k}\right\}$ denote the sequence of projections associated with the Schauder decomposition of $A\left(X_{s}\right)$. By [10, Theorem 6.2] this decomposition is monotone so that the decomposition constant $K_{2}$ of $A\left(X_{s}\right)$ is 1 . Hence for any $F=\Sigma_{k}^{*} p_{k}^{*}(F)$ in $A\left(X_{s}\right) *$,

$$
|F|_{1}^{* *} \geq \sup _{n}\left|\sum_{k \leq n} p_{k}^{*}(F)\right|_{1}^{* *}
$$

The reverse inequality being trivial, we obtain equality in $(A)$. Let now $\left\{F_{n}\right\}$ be a $\gamma$-Cauchy sequence in $C(X)_{s}$ where $F_{n}=\left\{J\left(x_{n, k}\right)\right\}, x_{n, k} \in X_{k}$. Then $\sup _{n}\left|F_{n}\right|_{1}^{*} *$ $=K<\infty$ and $\left|J\left(x_{n, k}\right)-J\left(x_{m, k}\right)\right|_{1}^{*} \rightarrow 0$ as $n, m \rightarrow \infty, k=1,2, \ldots$ Since $J\left(X_{k}\right)$ is complete, there exists $y_{k}$ in $X_{k}$ such that $J\left(x_{n, k}\right) \rightarrow J\left(y_{k}\right), k=1,2, \ldots$ Let $f \in A\left(X_{s}\right)$ with $|f|_{1}^{*}=1$. For any arbitrary but fixed $m$,

$$
\begin{aligned}
& \left|\sum_{b \leq n} J\left(y_{k}\right) f\right|=\lim _{n}\left|\sum_{k \leq n} J\left(x_{n, k}\right) f\right| \\
& \quad \leq \frac{\lim }{n}\left|\sum_{k \leq m} J\left(x_{n, k}\right)\right|_{1}^{* *} \leq \underset{n}{\leq \lim \inf \left|F_{n}\right|_{1}^{* *} \leq K}
\end{aligned}
$$


so that, by Theorem 3.1, $\left\{J\left(y_{k}\right)\right\} \in C(X)$. Hence $C(X)_{s}$ is $\gamma$-complete and this proof may also be used to show that it is normal.

The map $i$ is obviously well defined. It is $1-1$ since $\left\{X_{j}\right\}$ is a Schauder decomposition of $X$. That it is an isometry with respect to $\|_{1}$ and $\|_{2}$ is a consequence of the normality of $X_{s}$ and the fact that $\Sigma_{k} x_{k}=x$ in $X$ whenever $\Sigma_{k} J\left(x_{k}\right)=J(x)$ in $A\left(X_{s}\right) *$ and conversely.

Finally for $\left\{J\left(x_{k}\right)\right\} \in C(X), \Sigma_{k \leq n} J\left(x_{k}\right) \in i(X)$ and $\left\{\Sigma_{k \leq n} J\left(x_{k}\right)\right\} \stackrel{\gamma}{\rightarrow}\left\{J\left(x_{k}\right)\right\}$. Hence $i\left(X_{s}\right)$ is $\gamma$-dense in $C(X)_{s}$ and the proof is complete.

We note that if $\left\{X_{j}\right\}$ is nonmonotone, $i$ reduces to a linear isomorphism with respect to both norms.

As an illustration of this last theorem, observe that the unit vectors $\left\{e_{n}\right\}$, $e_{n}=\left\{\delta_{n, j}\right\}$, form a nonboundedly complete basis for $c_{0}$ so that $c_{0 s}$ is not $\gamma$-complete. Its completion is $m_{s}=\left(m, \sup _{n}\left|a_{n}\right|, \Sigma\left|a_{n}\right| / 2^{n}\right)$. Here, however, $m=l^{*}=$ $A\left(c_{0 s}\right) *$. We shall soon see that this is a characteristic property of bases in $B$ spaces.

We also note that $C(X)$ is isometrically isomorphic to the set of all sequences $\left\{x_{n}\right\}, x_{n} \in X_{n}$, such that $\sup _{n}\left|\Sigma_{k \leq n} x_{k}\right|_{1}<\infty$ with the norm (cf. [9])

$$
\left|\left\{x_{n}\right\}\right|=\sup _{n}\left|\sum_{k \leq n} x_{k}\right|_{1} .
$$

Definition 3.3. Let $X$ be a B-space with a (monotone) Schauder decomposition. The two-norm space $C(X)_{s}$ of Theorem 3.2 is called the canonical $\gamma$-completion of $X_{s}$. The space $C(X)$ is called the bounded completion of $X$.

Theorem 3.4. Let $\left\{X_{j}\right\}$ be a (monotone) Schauder decomposition of a B-space $X$. Then $C(X)$ is identical with $A\left(X_{s}\right)^{*}$ if and only if $X_{s}$ is k-reflexive.

Proof. Let $I$ be the identity map on $A\left(X_{s}\right)^{*}=\bigoplus \Sigma_{j}^{*} A\left(k-X_{s}\right)_{j}$ where $\left\{A\left(k-X_{s}\right)_{j}\right\}$ denotes the Schauder decomposition of $A\left(k-X_{s}\right)$. By definition, $C(X)=$ $\Sigma_{j}^{*} J\left(X_{j}\right)$, where $J\left(X_{j}\right) \subseteq A\left(k-X_{s}\right)_{j}\left[10\right.$, Theorem 4.4]. Hence $I: C(X) \rightarrow A\left(X_{s}\right)^{*}$. We thus have

$$
\begin{aligned}
I \text { is onto } & \Leftrightarrow \bigoplus \sum_{j}^{*} J\left(X_{j}\right)=\bigoplus \sum_{j}^{*} A\left(k-X_{s}\right)_{j} \\
& \Leftrightarrow J\left(X_{j}\right)=A\left(k \cdot X_{s}\right), \quad j=1,2, \cdots, \\
& \Leftrightarrow J: X_{s} \stackrel{\text { onto }}{\longrightarrow} k^{2} \cdot X_{s} \\
& \Leftrightarrow X_{s} \text { is } k \text {-reflexive }
\end{aligned}
$$

by [10, Theorem 4.7] and this concludes the proof.

Corollary 3.5. For a $B$-space $X$ with a basis, $X_{s}$ is $\gamma$-complete if and only if it is $\gamma$-reflexive. 
Proof. Every $\gamma$-reflexive space is obviously $\gamma$-complete. On the other hand, the $\gamma$-completeness of $X_{s}$ implies the boundedly completeness of the basis for $X$. Hence $X \cong C(X)=A\left(X_{s}\right) *$ by Theorem 3.4, that is $X_{s}$ is $\gamma$-reflexive.

Corollary 3.6 [9, p. 143]. Let $X$ be a B-space which bas a basis $\left\{x_{n}\right\}$ and let $Y=\left[y_{n}\right]$ be the closed linear subspace of $X_{1}^{*}$ spanned by the $y_{n}, y_{n}\left(x_{j}\right)=$ $\delta_{n, j}$. Then $Y^{*}$ is isomorphic to the B-space of scalars

$$
E=\left\{\left\{b_{n}\right\}: \sup _{n}\left|\sum_{k \leq n} b_{k} x_{k}\right|_{1}<\infty\right\}
$$

where the norm is defined by $\left\|\left\{b_{n}\right\}\right\|=\sup _{n}\left|\Sigma_{k \leq n} b_{k} x_{k}\right|_{1}$.

Proof. This follows from our remarks made earlier and the fact that the $\gamma$ completion of $X_{s}$ in this case is $Y^{*}=A\left(X_{s}\right)^{*}$.

Corollary 3.7. Let $X$ be a B-space with a (monotone) shrinking Schauder decomposition $\left\{X_{j}\right\}$. Then $X_{1}^{* *}$ is isomorphic (respectively isometrically isomorphic to) to $C(X)$ if and only if each $X_{j}$ is reflexive.

Proof. By [10, Corollary 3.5], $A\left(X_{s}\right)=X_{1}^{*}$ so that $A\left(X_{s}\right) *=X_{1}^{* *}$ and the conclusion now follows from Theorem 3.4 .

We may remark that if each $X_{j}$ is of dimension 1 , Corollary 3.7 reduces to a result of $\mathrm{R}$. C. James (see [9, p. 143]).

Theorem 3.8. Let $\left(X, \|_{1}\right)$ be a B-space with a Schauder decomposition $\left\{X_{j}\right\}$. If $i$ is defined as in Theorem 3.2, then $i(X)=\left\{\left\{J\left(x_{j}\right)\right\}: \Sigma_{j} J\left(x_{j}\right)\right.$ converges in $\left.A\left(X_{s}\right) *\right\}$. Further $\left\{X_{j}\right\}$ is boundedly complete if and only if $i$ is bijective, that is, $i(X)=C(X)$.

Proof. For $x=\Sigma_{j} x_{j}$ in $X, i(x)=\left\{J\left(x_{j}\right)\right\}$ and $\Sigma_{j} J\left(x_{j}\right)$ converges in $A\left(k-X_{s}\right)$, hence in $A\left(X_{s}\right) *$. Conversely, if $\left\{J\left(y_{j}\right)\right\} \in C(X)$ such that $\Sigma_{j} J\left(y_{j}\right)$ converges, then $\Sigma_{j} J\left(y_{j}\right) \in\left[\bigcup_{j} J\left(X_{j}\right)\right]=J(X)$, where [] denotes closed linear span. Thus for some $y$ in $X, J(y)=\Sigma_{j} J\left(y_{j}\right)$ and $i(y)=\left\{J\left(y_{j}\right)\right\}$.

If $\left\{X_{j}\right\}$ is boundedly complete for $X$, then so is $\left\{J\left(X_{j}\right)\right\}$ for $J(X)$. Hence

$$
\begin{aligned}
\left\{J\left(x_{j}\right)\right\} \in C(X) & \Leftrightarrow \sup _{n}\left|\sum_{j \leq n} J\left(x_{j}\right)\right|_{1}^{* *}<\infty \Leftrightarrow \sum_{j} J\left(x_{j}\right) \text { converges } \\
& \Leftrightarrow\left\{J\left(x_{j}\right)\right\} \in i(X) \Leftrightarrow C(X)=i(X) .
\end{aligned}
$$

The following result was observed in [10]. 
Theorem 3.9. Let $\left\{X_{j}\right\}$ be a monotone Scbauder decomposition for a B-space $X$. The canonical two-norm space $X_{s}$ is $\gamma$-reflexive if and only if $X_{s}$ is $k$-reflexive and $\gamma$-complete.

Proof. $X_{s}$ is $\gamma$-reflexive $\Leftrightarrow J: X \rightarrow A\left(X_{s}\right) *$ is $1-1$ and onto $\Leftrightarrow i$ is bijective and $A\left(X_{s}\right) *=C(X) \Leftrightarrow X_{s}$ is $k$-reflexive and $\left\{X_{j}\right\}$ is boundedly complete by Theorems 3.4 and 3.8 .

4.0 Pseudo-reflexivity. Let $\left(X, \|_{1}\right)$ be a $B$-space and $Y$ a closed linear subspace of $X_{1}^{*}$. Following Singer [9], we shall say that $X$ is $Y$-pseudo reflexive if the canonical map $J$ from $X$ into $Y^{*}$ is a linear isomorphism onto $Y^{*}$. If $J$ is an isometry as well, $X$ will be called $Y$-reflexive. Singer has shown [9, p. 140] that a necessary and sufficient condition for $X$ to be $Y$-reflexive is that the closed unit ball $S$ of $X$ be complete for the topology $\sigma(X, Y)$ and that $Y$ be an $X$-total subspace of $X_{1}^{*}$.

Let us observe that given a total, norming and closed linear subspace $Y$ of $X_{1}^{*}$, if there exists a norm $\|_{2}$ on $X$ such that $A\left(X_{s}\right)=Y$, then $X$ is $Y$-reflexive if and only if $X_{s}$ is $\gamma$-reflexive. In general, however, such a norm need not exist. If $Y$ is separable, a suitable second norm may be defined on $X$ such that for the resulting two-norm space $X_{s}$ we have $A\left(X_{s}\right)=Y$ [3. p. 124]. In this case we can give a different proof of the following theorem of Singer [9, p. 142]:

Theorem. Let $\left(X, \|_{1}\right)$ be a $B$-space and $Y$ a separable linear subspace of $X_{1}^{*}$. Then $X$ is $Y$-reflexive if and only if $S$, the closed unit ball of $X$, is sequentially complete for the topology $\sigma(X, Y)$ and $Y$ is an $X$-total subspace of $X_{1}^{*}$.

Proof. The necessity is clear and it is also obvious that $Y$ must be norming. On the other hand, to show that these conditions are sufficient, let $|x|_{2}=$ $\Sigma_{n}\left|f_{n}(x)\right| / 2^{n}$ where $\left\{f_{n}\right\}$ is dense in $\left\{f \in Y:|f|_{1}^{*}=1\right\}$. Then [3, p. 124], $X_{s}$ is normal, $\gamma$-precompact and $A\left(X_{s}\right)=Y$. Convergence in $\|_{2}$ being equivalent to convergence of each of the sequences $\left\{f_{n}().\right\}, n=1,2, \ldots$, the sequential completeness of $S$ for the topology $\sigma(X, Y)$ implies that $X_{s}$ is $\gamma$-complete. Hence $X_{s}$ is $\gamma$-compact whence it is $\gamma$-reflexive $[2, \$ 5.2]$. Hence $X$ is $Y$-reflexive.

We now investigate the $Y$-reflexivity of a $B$-space $\left(X,||_{1}\right)$ with a Schauder decomposition $\left\{X_{j}\right\}$, where $Y=A\left(X_{s}\right)$ and $X_{s}$ is the canonical two-norm space of $X$. The following theorem characterizes the $A\left(X_{s}\right)$-reflexivity of $X$.

Theorem 4.1. Let $X$ be a B-space with a (monotone) Schauder decomposition $\left\{X_{j}\right\}$ and let $X_{s}$ be the canonical two-norm space of $X$. The following are equivalent:

(1) $X$ is $A\left(X_{s}\right)$-pseudo reflexive (respectively $A\left(X_{s}\right)$-reflexive). 
(2) $X_{s}$ is $\gamma$-semi reflexive (respectively $\gamma$-reflexive).

(3) $\left\{X_{j}\right\}$ is boundedly complete and $X_{j}$ is reflexive for each $j$.

(4) $A\left(X_{s}\right) *=\Sigma_{j} J\left(X_{j}\right)=\Sigma_{j} A\left(k-X_{s}\right)_{j}$.

(5) $\left\{A\left(k-X_{s}\right)_{j}\right\}$ is shrinking for $A\left(X_{s}\right) *$ and $X_{j}$ is reflexive for each $j$.

(6) $X_{1}^{* *}=J(X) \oplus A\left(X_{s}\right)^{\perp}$, where $A\left(X_{s}\right)^{\perp}$ is the set $\left\{F \in X_{1}^{* *}: F\left(A\left(X_{s}\right)\right)=0\right\}$.

Proof. (1) $\Leftrightarrow$ (2) by definition, (2) $\Leftrightarrow$ (3) by Theorems 3.9, [10, Theorems 3.6, 4.7]. On the other hand $X_{s}$ is $y$-reflexive $\Leftrightarrow A\left(X_{s}\right) *=J(X)=\Sigma_{j} J\left(X_{j}\right) \subseteq$ $\Sigma_{j} A\left(k-X_{s}\right)_{j}=A\left(k-X_{s}\right) \subseteq A\left(X_{s}\right) *$ whence (2) $\Leftrightarrow(4)$. Furthermore, (4) $\Leftrightarrow A\left(X_{s}\right) *=$ $\Sigma_{j} A\left(k-X_{s}\right)_{j}$ and $J\left(X_{j}\right)=A\left(k-X_{s}\right)$ for every $j$ and this is equivalent to (5). Finally, the equivalence of (1) and (6) is a special case of the following theorem.

Theorem 4.2. Let $X_{s}=\left(X,\left\|_{1},\right\|_{2}\right)$ be a normal two-norm space satisfying $\left(n_{0}\right)$ where $\|_{2}$ is a $B_{0}^{*}$-norm. Then $X_{s}$ is $\gamma$-reflexive if and only if $X_{1}^{* *}=J(X)$ $\oplus A\left(X_{s}\right)^{\perp}$, where $A\left(X_{s}\right)^{\perp}$ is the annibilator of $A\left(X_{s}\right)$ in $X_{1}^{* *}$.

Proof. If $X_{s}$ is $\gamma$-reflexive, then $\left(A\left(X_{s}\right), \|_{1}^{*}\right) *=J(X) \cong X$. Hence $X_{1}^{* *}$ is isometrically isomorphic to the third conjugate of $A\left(X_{s}\right)$. Hence by a theorem of Diximier (see for instance $[4, \mathrm{p} .426]$ ), the projection

$$
\pi: X_{1}^{* *} \rightarrow J(X)
$$

defined by $\pi(F)=\left.F\right|_{J(X)}$ for $F$ in $X_{1}^{* *}$ is continuous with norm 1 and kernel $A\left(X_{s}\right)^{\perp}$. Hence $X_{1}^{* *}=J(X) \oplus A\left(X_{s}\right)^{\perp}$.

Conversely, since $X_{s}$ satisfies $\left(n_{0}\right), A\left(X_{s}\right)$ is a closed subspace of $X_{1}^{*}$. Therefore, given $f \in A\left(X_{s}\right) *$, by the Hahn-Banach theorem there exists $F \in X_{1}^{* *}$ with the same norm as that of $f$ and such that $\left.F\right|_{A\left(X_{s}\right)}=f$. Hence there exist $x \in X$ and $g \in A\left(X_{s}\right)^{\perp}$ such that $F=J(x)+g$. Now for any $\xi$ in $A\left(X_{s}\right)$ we have $F(\xi)=J(x)(\xi)$, that is, $f(\xi)=J(x)(\xi)$. Thus $f \equiv J(x)$ on $A\left(X_{s}\right)$ so that we may conclude $A\left(X_{s}\right) * \subseteq J(X)$. Since the other inclusion is obvious, we have $A\left(X_{s}\right)^{*}=$ $J(X)$, that is $X_{s}$ is $\gamma$-reflexive.

5.0 Direct sum of $B$-spaces. We now consider $B$-spaces $X$ which arise as a countable direct sum of other $B$-spaces $\left(X_{n}\right)$. In our case the structure of $X$ and its norm will be determined by an underlying space $B$, a $B K$ space. Many sequence spaces may be considered as examples of such spaces. Several such spaces have also been used as counterexample spaces by many authors.

The spaces $X_{n}$ may be considered as imbedded in $X$, hence treated as subspaces of $X$. In general, they do not form a decomposition of $X$ for norm convergence. Nevertheless, each $x$ in $X$ shall correspond to a unique sequence $\left\{x_{n}\right\}$, $x_{n} \in X_{n}$. Also $\left\{X_{n}\right\}$ will form a 'generalized decomposition' of $X$ in the sense $\bigcup_{k} P_{k}^{*}\left(X^{*}\right)$ shall be $X$-total in $X^{*}$, where $P_{k}: X \rightarrow X_{k}$. We shall see that a natural two-norm space $X_{s}$ may be defined as before and the usual arguments of two-norm 
spaces in Schauder decompositions adapted to spaces of this kind.

As an example, consider the space $(m)$. Here each $X_{n}=\mathbf{R}$ and the space $B$ is $(m)$ itself. The vectors $\left(e_{n}\right)$ form a generalized basis of $(m)$. A natural extension of the definition of boundedly complete bases shows that $\left(e_{n}\right)$ is boundedly complete for $(m)$. Note also that the associated two-norm space $\left(m_{s}\right)$ is $\gamma$-complete. We shall observe later that this is a special case of the analogue of [10, Theorem 3.6].

We begin with

Definition 5.1. A $B K$ space $\left(B,||_{B}\right)$ is a Banach space of real sequences such that $\left|\left\{a_{n}\right\}\right|_{B} \geq \Sigma_{n}\left|a_{n}\right| /\left(2^{n}\left(1+\left|a_{n}\right|\right)\right)$ for elements $\left\{a_{n}\right\}$ in $B$. The space $B$ is called normal if for any two sequences $\left\{a_{n}\right\}$ and $\left\{b_{n}\right\},\left|a_{n}\right| \leq\left|b_{n}\right|$ and $\left\{b_{n}\right\} \in B$ $\Rightarrow\left\{a_{n}\right\} \in B$. We say $B$ is ordered if for any two elements $\left\{a_{n}\right\},\left\{b_{n}\right\}$ in $B$ with $\left|a_{n}\right| \leq\left|b_{n}\right|$, we have $\left|\left\{a_{n}\right\}\right|_{B} \leq\left|\left\{b_{n}\right\}\right|_{B}$.

The above definition is equivalent to the usual definition of a $B K$ space since it implies (and is implied by) the fact that the coordinate functionals are continuous.

All $B K$ spaces $B$ considered in this paper are assumed to have the following properties:

(1) $B$ is ordered and normal,

(2) $B$ contains the unit vectors $\left\{e_{n}=\left\{\delta_{n, j}\right\}\right\}$ and $\left|e_{n}\right|_{B}=1$ for all $n$, and

(3) if $a=\left\{a_{n}\right\} \in B$, then $|a|_{B}=\sup _{n}\left\{|| a_{j}\left|\epsilon_{j n}\right|_{B}\right\}$ where $\epsilon_{j n}=1$ for $j \leq n$, = 0 for $j>n$.

This last postulate has the advantage of making \|\|$_{B}$ "monotone". In most cases of interest, the natural norm on the $B K$ space is either equal or equivalent to a norm introduced in the above manner.

Definition 5.2. Let $\left(X_{n},||_{n}\right), n=1,2, \ldots$, be a sequence of $B$-spaces and $\left(B, \|_{B}\right)$ a $B K$ space. By the direct sum with respect to $B$ of $\left(X_{n}\right)$, we shall mean the set of all those sequences $x=\left\{x_{n}\right\}$ with $x_{n} \in X_{n}$ and such that $\left\{\left|x_{n}\right|_{n}\right\}$ $\epsilon B$. This set will be denoted by $B-\Sigma_{n} X_{n}$, and when there is no cause for confusion about the underlying space $B$, merely by $\Sigma_{n} X_{n}$.

Theorem 5.3. Let $X=B-\Sigma_{n} X_{n}$ and for $x=\left\{x_{n}\right\} \in X$, define $\|x\|=\left|\left\{\left|x_{n}\right|_{n}\right\}\right|_{B}$. Then $(X,\|\|)$ is a $B$-space.

Proof. We need only show that $(X,\|\|)$ is complete. Let $\left\{x_{n}\right\}$ be a Cauchy sequence in $X$ where $x_{n}=\left\{x_{n}^{(j)}\right\}_{j=1}^{\infty}$. Then given $\epsilon>0, \exists N(\epsilon)$ such that $n, m>N$ implies

$$
\left\|x_{n}-x_{m}\right\|=\sup _{p}\left|\left\{\left|x_{n}^{(j)}-x_{m}^{(j)}\right|_{j} \epsilon_{j p}\right\}\right|_{B}<\epsilon .
$$

Since $B$ is ordered, for $n, m>N$ and any $j$, 


$$
\begin{aligned}
\left|x_{n}^{(j)}-x_{m}^{(j)}\right|_{j} & =\left|\left\{\left|x_{n}^{(k)}-x_{m}^{(k)}\right|_{k} \delta_{j, k}\right\}\right|_{B} \\
& \leq\left|\left\{\left|x_{n}^{(k)}-x_{m}^{(k)}\right|_{k} \epsilon_{k j}\right\}\right|_{B}<\epsilon .
\end{aligned}
$$

Hence $\left\{x_{n}^{(j)}\right\}$ is Cauchy in $X_{j}$ for each $j$, so that $x_{n}^{(j)} \rightarrow x_{0}^{(j)} \in X_{j}$ uniformly for all $j$. Since $\left\{\left|x_{n}^{(j)}\right|_{j}\right\} \in B$ for each $n$ and $B$ is ordered, it follows that $\left\{\left|x_{n}^{(j)}\right|_{j}\right\}_{n=1}^{\infty}$

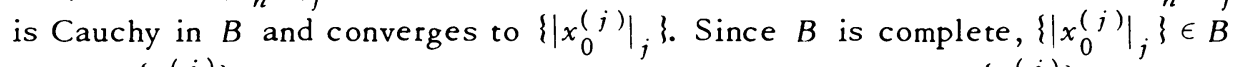
whence $\left\{x_{0}^{(j)}\right\} \in X$. It is routine to show that $x_{n}$ converges to $\left\{x_{0}^{(j)}\right\}$ and this completes the proof.

Consider the subspace $Y_{n} \subseteq X$ consisting of all elements of the form $\left\{x_{n} \delta_{n, j}\right\}$, $\delta_{n, j}$ being the Kronecker $\delta$. The map $t_{n}$ from $X_{n}$ onto $Y_{n}$ defined by $t_{n}(z)=$ $\left\{z \delta_{n, j}\right\}, z \in X_{n}$, is clearly an isometry. We shall, therefore, occasionally identify $X_{n}$ and $Y_{n}$ in the future and consider $X_{n}$ as a subspace of $X$.

Definition 5.4. Let $X=B-\Sigma_{n} X_{n}$. The canonical two-norm space of $X$ is the two-norm space $\left(X,\|\|,\|\|_{2}\right)$ where, for $x=\left\{x_{n}\right\} \in X,\|x\|_{2}=\Sigma_{n}\left|x_{n}\right|_{n^{\prime}} / 2^{n}$.

Theorem 5.5. Let $X_{s}$ be the canonical two-norm space of $X=B-\Sigma_{n} X_{n}$. Then $X_{s}$ is normal and satisfies $\left(n_{0}\right)$.

Proof. Let $x_{n} \stackrel{\gamma}{\rightarrow} \xi$ in $X_{s}$ where $x_{n}=\left\{x_{n, j}\right\}_{j=1}^{\infty}, \xi=\left\{\xi_{j}\right\}, x_{n, j}, \xi_{j} \in X_{j}, j=$ $1,2, \ldots$. For each $p$,

$$
\begin{aligned}
\left|\left\{\left|\xi_{j}\right|_{j} \epsilon_{j p}\right\}\right|_{B} & =\frac{\left.\left.\left|\sum_{j=1}^{\prime}\right| \xi_{j}\right|_{j} e_{j}\right|_{B}}{\left|\lim _{n}\left(\sum_{j=1}^{p}\left|x_{n, j}\right|_{j} e_{j}\right)\right|_{B}} \\
& =\left.\left.\lim _{n}\left|\sum_{j=1}^{p}\right| x_{n, j}\right|_{j} e_{j}\right|_{B} \leq \liminf _{n}\left\{\left\|x_{n}\right\|\right\} .
\end{aligned}
$$

It follows that $\|\xi\| \leq \lim \inf _{n}\left\|x_{n}\right\|$ showing that $X_{s}$ is normal. That $X_{s}$ satisfies $\left(n_{0}\right)$ is clear since, for any $x$ in $X,\left|x_{j}\right|_{j} \leq\|x\|\left(B\right.$ is ordered) whence $\|x\|_{2} \leq$ $\|x\|$.

An immediate corollary of the above theorem is that the canonical map $J: X$ $\rightarrow A\left(X_{s}\right)^{*}$ is an isometry. In what follows we write $X_{1}^{*}$ for $(X,\|\|)^{*}$. We shall have no occasion to consider the space $\left(X_{1}, \|_{1}\right) *$ and once again the reader is asked not to confuse the latter space for $X_{1}^{*}$.

Definition 5.6. Let $X=B-\Sigma_{n} X_{n}$. The sequence $\left(X_{n}\right)$ is said to be boundedly complete if for any sequence $\left\{x_{n}\right\}, x_{n} \in X_{n}, \sup _{n}\left|\left\{\left|x_{j}\right|_{j} \epsilon_{j n}\right\}\right|_{B}<\infty$ implies $\left\{x_{n}\right\} \in X$. 
Definition 5.7. Let $X=B-\Sigma_{n} X_{n}$ and let $Z_{n}$ be the subspace of $X$ consisting of sequences whose first $n$ coordinates are zeros. For $f \in X_{1}^{*}$ let $|f|_{1, n}^{*}=$ $\sup \left\{|f(x)|: x \in Z_{n},\|x\|=1\right\}$. Then $\left(X_{n}\right)$ is said to be shrinking for $f \in X_{1}^{*}$ if $\lim _{n}|f|_{1, n}^{*}=0$.

In the special case when $\left(Y_{n}\right)$ forms a Schauder decomposition of $X$, the above definitions reduce to the usual definitions of boundedly completeness and the shrinking property. We also note that under this definition the unit vectors form a generalized boundedly complete basis for $(m)$ and are shrinking for every element in $l$. This is a special case of the following

Theorem 5.8. Let $X=B-\Sigma_{n} X_{n}$. Then the sequence $\left(X_{n}\right)$ is boundedly complete if and only if $X_{s}$ is $\gamma$-complete. Further $\left(X_{n}\right)$ is shrinking for $f \in X_{1}^{*}$ if and only if $f \in A\left(X_{s}\right)$.

The proof is similar to those of $[10$, Theorems $3.4,3.6]$. We indicate the lines of the proof of the second assertion leaving that of the first to the reader.

Proof of second assertion. Given $\epsilon>0$ and $f \in A\left(X_{s}\right)$ we can find $\delta>0$ such that $\|x\| \leq 1$ and $\|x\|_{2}<\delta$ imply $|f(x)|<\epsilon$. Choose $N>0$ such that $n>N$ implies $\Sigma_{j>n} 2^{-j}<\delta$. Then for $\xi=\left\{\xi_{j}\right\}$ in $Z_{n}$ with $\|\xi\|=1$ we have $\|\xi\|_{2}<\delta$ so that $|f(\xi)|<\epsilon$. This shows $|f|_{1, n}^{*}<\epsilon$ for $n>N$.

For the other part, we identify $x_{n, j} \in X_{j}$ with $\left\{x_{n, j} \delta_{j, p}\right\}_{p=1}^{\infty}$ in $Y_{j}, j=1,2$, $\cdots$ Let $f \in X_{1}^{*}, x_{n} \rightarrow 0(\gamma)$ in $X_{s}$ and suppose $\left\|x_{n}\right\| \leq 1$. Find $N>0$ such that $|f|_{1, N}^{*}<\epsilon / 2, \epsilon>0$ being arbitrary. There exists $M$ such that $\left|f\left(x_{n, j}\right)\right|<\epsilon / 2 N$ whenever $n>M$ and $1 \leq j \leq N$. Clearly for $n>M$ we have

$$
\left|f\left(x_{n}\right)\right| \leq\left|\sum_{j=1}^{N} f\left(x_{n, j}\right)\right|+\left|f\left(x_{n}-\sum_{j=1}^{N} x_{n, j}\right)\right|<N \cdot \frac{\epsilon}{2 N}+\frac{\epsilon}{2}=\epsilon
$$

showing that $f$ is $\gamma$-linear. This completes the proof.

For $x=\left\{x_{n}\right\} \in X$, the map $S_{n}: X \rightarrow X$ defined by $S_{n}(x)=\left\{x_{j} \epsilon_{j n}\right\}$ is clearly continuous. Hence the projection operator $P_{n}: X \rightarrow Y_{n}, P_{n}(x)=\left\{x_{j} \delta_{j, n}\right\}$ is also continuous. Since $P_{n}^{*}\left(X_{1}^{*}\right) \subseteq A\left(X_{s}\right)$ trivially and $A\left(X_{s}\right)$ is closed, $\bigoplus \Sigma_{k=1}^{\infty} P_{k}^{*}\left(X_{1}^{*}\right) \subseteq A\left(X_{s}\right)$. On the other hand for any $f$ in $A\left(X_{s}\right),|f|_{1, n}^{*} \rightarrow 0$ by the last theorem so that $\left|f-\Sigma_{k \leq n} P_{k}^{*}(f)\right|_{1}^{*} \rightarrow 0$ as $n \rightarrow \infty$. From these considerations we get

Theorem 5.9. Let $X=B-\Sigma_{n} X_{n}, X_{s}$ its canonical two-norm space. Then $\left\{P_{k}^{*}\left(X_{1}^{*}\right)\right\}$ is a Schauder decomposition of $A\left(X_{s}\right)$.

6.0 The $\gamma$-completion of $X_{s}, X=B-\Sigma_{n} X_{n}$. As in the case of $B$-spaces with Schauder decompositions, the $\gamma$-completion of the canonical two-norm space $X_{s}$ where $X=B-\Sigma_{n} X_{n}$ may be constructed. One easily verifies that in this case 
the space $C(X)$ may be identified with the space $B-\Sigma_{n}^{\prime} X_{n}$ of all sequences $\left\{x_{n}\right\}$, $x_{n} \in X_{n}$, such that

$$
v\left(\left\{x_{n}\right\}\right)=\sup _{n}\left\{\left|\left\{\left|x_{j}\right|_{j} \epsilon_{j n}\right\}\right|_{i 3}\right\}
$$

is finite. The norm in the $B$-space $C(X)$ is $N()$. It is also possible to describe the space $C(X)$ in a different way.

The $F$-space $\left(s, \|_{s}\right)$ of all scalar sequences with the Fréchet metric $\| s$ has the unit vectors $\left\{e_{k}\right\}$ for a basis. Since $B$ is dense in $s,\left(B, \mid \|_{s}\right) *$ is the linear span $\left[e_{n}\right]$. It is clear that $B_{s}=\left(B,||_{B},||_{s}\right)$ is normal $\left(||_{B}\right.$ being 'monotone') so that $A\left(B_{s}\right)$ is the closure of $\left[e_{n}\right]$ in $\left(B,||_{B}\right) *\left[2\right.$, p. 277]. Hence $\left\{e_{n}\right\}$. is a basis for $A\left(B_{s}\right)$ and a weak-* basis for $A\left(B_{s}\right) *$. It is easily verified that $A\left(B_{s}\right)^{*}$ is an ordered, normal $B K$ space consisting of scalar sequences $\left\{a_{k}\right\}$ with $\left|\left\{a_{k}\right\}\right|_{0}=\sup _{n}\left\{\left|a_{k} \epsilon_{k n}\right|_{B}\right\}$ finite. But this is precisely the space $C(B)$ so that $C(B)=A\left(B_{s}\right)^{*},||_{0}$ being its norm. We now have

Theorem 6.1. $B-\Sigma_{n}^{\prime} X_{n}=C(B)-\Sigma_{n} X_{n}$.

Proof. We need only show that the set on the right is contained in the one on the left. The normality of $B_{s}$ shows that the canonical map $B \rightarrow A\left(B_{s}\right) *=$ $C(B)$ is an isometry. Let |||| $\mid$ denote the norm in $C(B)-\Sigma_{n} X_{n}$ and suppose $\left\{x_{k}\right\}$ is an element of it. Then,

$$
\begin{aligned}
\left\|\left\{x_{k} \epsilon_{k n}\right\} \mid\right\| & =\left|\left\{\left|x_{k}\right|_{k} \epsilon_{k n}\right\}\right|_{C(B)} \\
& =\left|\left\{\left|x_{k}\right|_{k} \epsilon_{k n}\right\}\right|_{B}=N\left(\left\{x_{k} \epsilon_{k n}\right\}\right),
\end{aligned}
$$

showing that $\left\{x_{k}\right\} \in B-\Sigma_{n}^{\prime} X_{n}$ and completing the proof.

7.0 The $\alpha$-duals. Given the space $X=B-\Sigma_{n} X_{n}$, we shall call the canonical two-norm space of the space $A\left(X_{s}\right)$ as the $\alpha$-dual of $X_{s}$ and denote it by $\alpha-X_{s}$. Higher $\alpha$-duals are defined by induction. We note that $\alpha^{2}-X_{s}$ coincides with the $k$-dual (see $\$ 1$ ) of $a-X_{s}$. In the special case when $\left(Y_{n}\right)$ forms a Schauder decomposition of $X$, the $\alpha$-duals of $X_{s}$ are identical with its $k$-duals.

It was shown in $\$ 6$ that for any $B K$ space $B, A\left(B_{s}\right)$ has the unit vectors $\left\{e_{k}\right\}$ for a basis. Thus one may define the $\alpha$-duals of $B_{s}$ (called $T\left(B_{s}\right), T^{2}\left(B_{s}\right)$ etc., in [6]). It was shown in [6] that in general $\alpha^{2}-B_{s}$ is isometrically imbedded in $B_{s}$ and that this isometry is onto $B_{s}$ if and only if $B$ has $\left\{e_{k}\right\}$ for a basis.

We shall show here that these results may be extended to our present setting. For $X=B-\Sigma_{n} X_{n}$, we call $X_{s}$ " $\alpha$-reflexive" if $\alpha^{2}-X_{s}$ is isometrically isomorphic to $X_{s}$ under the canonical map. We begin by showing

Theorem 7.1. Let $X=B-\Sigma_{n} X_{n}$. Then, if $X_{j}$ is reflexive for each $j, J^{-1}$ : $a^{2}-X_{s} \stackrel{\text { onto }}{\rightarrow} X_{s}$, where $J$ denotes the canonical map from $X_{s}$ into $A\left(X_{s}\right) *$. 
Proof. Let $\left\{A\left(X_{s}\right)_{k}, p_{k}\right\}$ be the Schauder decomposition of $A\left(X_{s}\right),\left\{A\left(X_{s}\right)_{k}^{*}, p_{k}^{*}\right\}$ the Schauder decomposition of $A\left(\alpha-X_{s}\right)$ in the norm topology of $A\left(X_{s}\right) *$. By the reflexivity of $X_{j}$ for each $j$, it follows that

$$
A\left(\alpha \cdot X_{s}\right)=\left[\bigcup_{j} A\left(X_{s}\right)_{j}^{*}\right]=\left[\bigcup_{j} J\left(Y_{j}\right)\right] .
$$

Since $J$ is an isometry from $X$ into $A\left(X_{s}\right)^{*}, J^{-1} A\left(\alpha-X_{s}\right)=\left[\bigcup_{j} Y_{j}\right] \subseteq X$, the closure in [] being with respect to the norm in $X$. This completes the proof.

Theorem 7.2. Let $X=B-\Sigma_{n} X_{n}$. If $B_{s}$ is a-reflexive then $\left(X_{n}\right)$ is a Schauder decomposition of $X$ in the sense that $X=\bigoplus \Sigma_{n} Y_{n}$. Further if $B_{s}$ is a-reflexive, $X_{j}$ is reflexive for each $j$ if and only if $X_{s}$ is a-reflexive.

Proof. Let $x=\left\{x_{n}\right\} \in X, x_{n} \in X_{n}$. Let $y_{n}=\left\{x_{j} \delta_{j, n}\right\}$. We shall show that $\left\|x-\Sigma_{j \leq n} y_{j}\right\| \rightarrow 0$. Indeed,

$$
\begin{aligned}
\left\|x-\sum_{j \leq n} y_{j}\right\| & =\sup _{m}\left\{\left.\left.\left|\sum_{j=n}^{m}\right| x_{j}\right|_{j} e_{j}\right|_{B}\right\} \\
& \leq\left.\left.\left|\sum_{j>n}\right| x_{j}\right|_{j} e_{j}\right|_{B} \rightarrow 0
\end{aligned}
$$

since by the $\alpha$-reflexivity of $B_{s},\left\{e_{j}\right\}$ is a basis for $B$ and $\left\{x_{n}\right\} \in X \Rightarrow\left\{\left|x_{n}\right|_{n}\right\} \epsilon$ $B \Rightarrow \Sigma_{n}\left|x_{n}\right|{ }_{n} e_{n}$ converges in $B$.

To show the other part, observe that $\left\{Y_{n}\right\}$ is a Schauder decomposition of $X$ implies that $\alpha$-reflexivity coincides with $k$-reflexivity. An application of $[10$, Theorem 4.7] completes the proof.

The following theorem characterizes the $\alpha$-reflexivity of $X_{s}$.

Theorem 7.3. Let $X=B-\Sigma_{n} X_{n}$. Then $X_{s}$ is a-reflexive if and only if $\left(X_{n}\right)$ forms a Schauder decomposition of $X$ and $X_{n}$ is reflexive for each $n$.

Proof. If $X_{s}$ is $\alpha$-reflexive, $J(X)=A\left(\alpha-X_{s}\right)$. Since $A\left(\alpha-X_{s}\right)$ has a Schauder decomposition $\left\{A\left(X_{s}\right)_{k}^{*}, p_{k}^{*}\right\}$, it follows that $A\left(X_{s}\right)_{k}^{*}=p_{k}^{*}(J(X))$ and $\left\{p_{k}^{*}(J(X)), p_{k}^{*}\right\}$ forms a Schauder decomposition for $J(X)$. But for any $k, p_{k}^{*}(J(X))=J\left(Y_{k}\right)$. For, if $f=\Sigma_{k} f_{k} \in A\left(X_{s}\right), x=\left\{x_{k}\right\} \in X$,

$$
\begin{aligned}
p_{k}^{*}(J(x)) f & =J(x) p_{k}(f)=f_{k}(x)=f_{k}\left(y_{k}\right) \\
& =J\left(y_{k}\right) f_{k}=J\left(y_{k}\right) f
\end{aligned}
$$

so that $p_{k}^{*}(J(x))=J\left(y_{k}\right), y_{k}=\left\{x_{j} \delta_{k, j}\right\}$, whence $p_{k}^{*}(J(X)) \subseteq J\left(Y_{k}\right)$. Since $p_{k}^{*}\left(J\left(y_{k}\right)\right)$ $=J\left(y_{k}\right)$, the other inclusion is clear and our assertion is proved. It follows then that $A\left(X_{s}\right)_{k}^{*}=J\left(Y_{k}\right)$ for every $k$, whence $Y_{k}$ is reflexive for every $k$, and $J(X)=$ $\bigoplus \Sigma_{k} J\left(Y_{k}\right)$, that is, $X=\bigoplus \Sigma_{k} Y_{k}$. 
The converse follows from [10, Theorem 4.7] and the fact that if $X=\bigoplus \Sigma_{k} Y_{k}$, then $\alpha$-reflexivity coincides with $k$-reflexivity. This completes the proof.

Corollary 7.4. Let $X=B-\Sigma_{n} X_{n}$. If $X_{s}$ is $\gamma$-complete and $\alpha$-reflexive, then $X_{s}$ is $\gamma$-reflexive.

Let us remark that the converse to the above corollary is false. One may take $X=(m)$. Here $m_{s}$ is $\gamma$-reflexive but not $\alpha$-reflexive. However, we have the following theorem.

Theorem 7.5 (cf. [8]). Let $X=B-\Sigma_{n} X_{n}$. The following are equivalent:

(1) $X$ is reflexive.

(2) $X_{s}$ is a-reflexive, $\left(X_{n}\right)$ is boundedly complete and $\left(X_{n}\right)$ is shrinking for $(X,\|\|)^{*}$.

(3) $X_{n}$ is reflexive for each $n,\left(X_{n}\right)$ is boundedly complete and $\left(X_{n}\right)$ is shrinking for $(X,\|\|)$.

Proof. $X$ is reflexive if and only if $X_{s}$ is $\gamma$-reflexive and saturated [2, Theorem 3.7]. Recall that $X_{s}$ is saturated if $A\left(X_{s}\right)=(X,\|\|)^{*}$. The equivalence of (1) and (3) now follows from Theorem 6.4 and Theorem 5.8. That (2) implies (1) is clear since $X=\bigoplus \Sigma_{n} X_{n}$ in this case, a-reflexivity is identical with $k$ reflexivity and the conclusion follows from [8, Theorem 2$]$. It remains to show that (1) implies (2).

The reflexivity of $X$ immediately implies the reflexivity of $Y_{n}$, hence that of $X_{n}$, for each $n$. As observed before, $X_{s}$ is $\gamma$-reflexive and saturated so that $\left(X_{n}\right)$ is boundedly complete and $\left(X_{n}\right)$ is shrinking for $(X,\|\|)^{*}$. To complete the proof, we must show that $X_{s}$ is $\alpha$-reflexive. Since $X$. is reflexive, $X_{n}$ is reflexive for each $n$ and $A\left(X_{s}\right)=(X,\|\|) *$, we have

$$
J(X)=(X,\|\|)^{* *}=A\left(X_{s}\right)^{*}=\sum_{\dot{k}}^{*} p_{k}^{*}\left(A\left(X_{s}\right)^{*}\right)=\sum_{k}^{*} J\left(Y_{k}\right)
$$

where $\Sigma^{*}$ denotes convergence in the weak-* topology of $(X,\|\|)^{* *}$. This means that $\left(Y_{k}\right)$ is a weak Schauder decomposition, and hence [7, I.20] a Schauder decomposition of $X$ in the norm topology. Hence $X_{s}$ is $\alpha$-reflexive by Theorem 7.3 completing the proof.

Corollary 7.6. If $X=B-\Sigma_{n} X_{n}$, where $\left(X_{n}\right)$ does not form a Schauder decomposition of $X$, then $X$ is not norm-reflexive. In particular, for any sequence $\left(X_{n}\right)$ of B-spaces, $m-\Sigma_{n} X_{n}$ is not reflexive.

8.0 Use as counterexamples. We conclude this paper with a remark on the usefulness of the spaces $X=B-\Sigma_{n} X_{n}$ as counterexamples. Day( $\left.{ }^{1}\right)$ gives examples of reflexive $B$-spaces not isomorphic to uniformly convex spaces. Here one takes $B=$

(1) Bull. Amer. Math. Soc. 47 (1941), 313-317. 
$l^{p}, 1<p<\infty$, and the spaces $X_{n}$ are finite dimensional spaces. If we take $B=l$ and $X_{n}=c_{0}$ for each $n$ (all over R), we obtain [10, Remark after Theorem 5.3] an example of a $B$-space with a boundedly complete Schauder decomposition but not isometrically isomorphic to a dual space (cf. [8]). Incidentally, this example also illustrates Theorem 7.2 since $l_{s}$ is known to be $\alpha$-reflexive. If one takes $B=l$ and $X_{n}=m$ for each $n$, one obtains an example of a nonseparable $B$-space with a Schauder decomposition. Finally, by taking $B=m, X_{n}=c_{0}$ for each $n$, one obtains $[6$, p. 113] an example of a $B$-space with a countable determing set (ihat is a countable set $D=\left\{f_{n}\right\} \subset\left\{f:|f|_{1}^{*} \leq 1\right\}$ such that $\|x\|=\sup \{|f(x)|: f \in D\},\|\|$ and ||$_{1}^{*}$ being the norms in the space and its conjugate respectively) which is neither separable nor the dual of a separable space. For other counterexamples concerning two-norm spaces, the reader is referred to [6].

\section{REFERENCES}

1. L. Alaoglu, Weak topologies of normed linear spaces, Ann. of Math. (2) 41 (1940), 252-267. MR 1, 241.

2. A. Alexiewicz and Z. Semadeni, The two-norm spaces and their conjugate spaces, Studia Math. 18 (1959), 275-293. MR 22 \#5878.

3. - Some properties of two-norm spaces and a characterization of reflexivity of Banach spaces, Studia Math. 19 (1960), 115-132。 MR 22 \#310.

4. G. Köthe, Topologische linear Räume. I, Die Grundlehren der math. Wissenschaften, Band 107, Springer-Verlag, Berlin, 1960; English transl., Die Grundlehren der math. Wissenschaften, Band 159, Springer-Verlag, New York, 1969. MR 24 \#A411; MR 40 \#1750.

5. W. Orlicz and V. Pták, Some remarks on Saks spaces, Studia Math. 16 (1957), 5668. MR $20 \# 1198$.

6. S. Rothman, Banach spaces via two-norm spaces, Dissertation, University of Wisconsin, Milwaukee, Wis., 1970.

7. W. H. Ruckle, The infinite sum of closed subspaces of an F-space, Duke Math. J. 31 (1964), 543-554. MR 29 \#3862.

8. B. L. Sanders, Decompositions and reflexivity in Banach spaces, Proc. Amer. Math. Soc. 16 (1965), 204-208. MR 30 \#2318.

9. I. Singer, On Banach spaces reflexive with respect to a linear subspace of the ir conjugate space. III, Rev. Math. Pures Appl. 8 (1963), 139-150. MR 27 \#2831.

10. P. K. Subramanian, Two-norm spaces and decompositions of Banach spaces. I, Studia Math. 33 (1972), 179-194.

DEPARTMENT OF MATHEMATICS, MISSOURI SOUTHERN COLLEGE, JOPLIN, MISSOURI 64801

DEPARTMENT OF MATHEMATICS, QUINNIPIAC COLLEGE, HAMDEN, CONNECTICUT 06518 